\title{
Interruptions and Geographic Challenges to Nurses' Cognitive Workload
}

\author{
Donna A. Redding, PhD, RN-BC; \\ Sherry Robinson, PhD, GCNS-BC
}

\begin{abstract}
The cognitive workload of nurses needs to be protected from interruptions as much as possible to prevent untoward patient outcomes. In this study, the type and frequency of work interruptions for nurses in medical-surgical units in a midwestern tertiary care medical center were identified. In addition, nurses' travel patterns were observed and recorded as they provided care. The intent was to identify methods for reducing interruptions and improving nurses' cognitive work efficiency. Key words: cognitive workload, interruptions, nurses, observation, patient safety
\end{abstract}

$\mathbf{T}$ HE FAST PACE of acute inpatient care requires nurses to constantly shift their attention to make clinical decisions and manage care for groups of patients in a continually changing environment. Nurses must integrate complex thinking processes with psychomotor and affective skills to deliver appropriate interventions. They must repeatedly revise their priorities as patients' conditions change. At the same time, nurses must juggle important patient education, family concerns, new admissions, discharges, and numerous other activities for other patients. ${ }^{1}$ This complex thinking is disrupted by multiple interruptions and distractions. These disruptions compete for nurses' attention and can lead to errors or omissions in care and possibly pose a patient safety risk. ${ }^{2}$

Over the past decade, there has been a growing emphasis on patient safety in hospitals. Both the Agency for Healthcare Research

\footnotetext{
Autbor Affiliations: Nursing Administration, Memorial Medical Center (Dr Redding) and Southern Illinois University School of Medicine (Dr Robinson), Springfield, Illinois.

The authors have no conflict of interest.

Corresponding Autbor: Donna A. Redding, PbD, $R N-B C$, Nursing Administration, Memorial Medical Center, $701 \mathrm{~N}$ First St, Springfield, IL 62781 (redding. donna@mbsil.com).

Accepted for publication: January 3, 2009
}

and Quality and The Joint Commission have established goals for patient safety. ${ }^{3,4}$ Experts in patient safety indicate that interruptions and distractions can have a profound impact on patient safety. These distractions may be more related to systems design flaws than to individual performance. An understanding of these interruptions in the acute care environment can be attained only by observing nurses while they are actually caring for patients. This understanding will provide direction to redesign systems and environments to enhance patient safety and improve nurse satisfaction. ${ }^{5-8}$

The purpose of this study was to examine the type and frequency of work interruptions for nurses in medical-surgical hospital units in a midwestern urban tertiary care hospital. The intent was to identify possible methods for either reducing interruptions or improving the efficiency of nurses' cognitive work. The study was designed to replicate the work of Potter et $\mathrm{al}^{9,10}$ to record nurses' trajectory patterns as they went about their work and the nature and number of interruptions to that work.

\section{LITERATURE REVIEW}

To study interruptions, researchers have shadowed nurses and observed them as they 
cared for patients throughout their shifts. ${ }^{6,9-12}$ For those studies that reported number of interruptions, the number ranged from 4 interruptions per hour ${ }^{10}$ to 6 per hour. ${ }^{6}$ Nurses were interrupted by patients, family members, physicians, nursing technicians, other nurses, students, and staff from other departments. Nurses were interrupted with telephone calls, pagers, and face-to-face interruptions. Some of the interruptions originated with problems from the system such as redundant pages and messages. ${ }^{6,9-12}$ Each interruption reflected an operational loss and was estimated to cost $\$ 95 .{ }^{11}$

Many of these interruptions occurred during the intervention phase of the nursing process. Nurses were in the process of performing such activities as traveling to patient rooms, gathering supplies, preparing or administering medication, and patient teaching. . $^{2,10,13,14}$ These interruptions created a risk for medication error and the potential for unintentional omissions in care.

Researchers expressed much concern about interruptions during medication preparation and administration. ${ }^{9,10}$ They noted that nurses who prepared medications for all of their patients and then delivered the medications from room to room experienced fewer interruptions than did those who prepared and delivered the medication individually. ${ }^{15}$ One researcher suggested a visual signal such as a hat or apron with the words "Please do not interrupt" for the nurse to wear when preparing medications or when dealing with a crisis. ${ }^{12}$ Following this suggestion, Pape et $\mathrm{al}^{16}$ examined the effect of a "Do Not Disturb" sign placed above automated medication dispensing machines and medication carts. Use of the sign resulted in a decrease in interruptions by physicians, other nurses, other personnel, and visitors. The greatest decrease occurred in interruptions from other nurses and the smallest decrease was in the interruptions from physicians.

The literature supports the fact that interruptions are common. There is a documented need for hospitals to examine closely the specific type of interruptions experienced by their nurses, determine if there are patterns to those interruptions, and make system changes to reduce interruptions.

\section{METHODS}

\section{Purpose}

This study was designed to examine the nature of workload interruptions for nurses working in medical-surgical hospital units in a midwestern urban tertiary care hospital, with the intent to identify methods for reducing interruptions and improving the nurses' cognitive work efficiency. The study replicated the work of Potter et al $^{9,10}$ to record nurses' trajectory patterns while they worked and the nature and number of interruptions to that work.

\section{Setting}

The study was conducted in medicalsurgical nursing units at a 562-bed tertiary care hospital in the midwest. The study was approved by the local institutional review board.

\section{Design}

The descriptive study included quantitative and qualitative observation techniques. Development of data collection tools began with observations by 2 doctorally prepared nurse faculty practitioners. These individuals observed 2 nurses each over 2 (2-hour) time periods. The observers carried unit diagrams on which they recorded nurses' travel patterns during patient care. They also kept detailed field notes on nurses' actions and conversations with patients, families, and other caregivers. Types of interruptions were identified from these observations, and a tally sheet of interruptions was developed. The unit diagrams and tally sheets were then utilized by senior nursing students to collect the study data under the observation of another researcher.

\section{Sample}

A convenience sample of 32 staff nurses was observed in the study during the day shift, working in 6 medical-surgical nursing units. 
The nurses were asked to allow nursing student data collectors to observe their work for 1-hour time periods during active involvement in patient care. Each nurse signed an informed consent form.

The units comprised 2 medical units, 2 surgical units, and 2 cardiology units ( 1 medical, 1 surgical). The units ranged in size from 30 beds (medical oncology) to 37 beds (cardiology surgical). Three of the units (medical, surgical, and cardiology medical) included intermediate care (IMC) beds with invasive monitoring capacity. The IMC units had a higher care hour per patient day and a slightly higher nurse-to-patient ratio than the other units because of patient acuity. Patient length of stay varied from a single-day stay in the nonIMC surgical unit to several weeks in the medical unit.

\section{Data collection}

The nursing student data collectors completed local human subjects' research education as part of their preparation for participation. They were educated in use of the interruptions tally sheet and unit diagram forms by the researcher. To maximize interrater reliability, the researcher coached the students in standardized documentation of observations and continually observed their data collection. Each student was required to practice with the tools prior to actual data collection. Students were instructed to have no conversation with the nurses during observation but rather to strictly observe in silence. They were further instructed not to enter patient rooms with the subjects to avoid creating another interruption. Instead, they listened for activity and nurse-patient communication from the patient's room doorway. They used the interruptions tally sheet to mark observed interruptions and the unit diagram form to trace nurses' travel patterns during patient care.

\section{Analysis}

Data were analyzed in aggregate across units by the students and the researcher using descriptive statistics. Interruptions frequen- cies were calculated by type. Subjects' travel patterns were qualitatively assessed and discussed to identify possible sources of interruptions and cumbersome processes interfering with patient care.

\section{FINDINGS}

\section{Major themes of interruptions}

A total of 244 interruptions were recorded (Table 1). Six major themes were reflected in the data: (1) employees asking questions, (2) distracting peripheral conversations, (3) supplies not on hand requiring the nurse to go elsewhere to acquire them, (4) phone calls, (5) family questions, and (6) patient call lights.

The major source of interruptions was questions from other caregivers. Employees asked for assistance, offered help, requested patient information, initiated personal conversations, and asked for clarification. To maintain patient confidentiality, some nursing technicians approached the nurse directly rather than using electronic communication. Several employees interrupted the nurse for information readily attainable from the medical record.

The second highest category was distracting peripheral conversations. These included conversations among employees, physicians, or visitors that did not actually include the nurse as a participant.

Table 1. Types and frequency of observed interruptions

\begin{tabular}{|lc|}
\hline Type of interruption & $\boldsymbol{n}(\%)$ \\
\hline Employee asking question face & $76(31.2)$ \\
$\quad$ to face & $55(22.5)$ \\
Distracting peripheral & \\
$\quad$ conversation & $19(7.8)$ \\
Supplies not on hand & $19(7.8)$ \\
Telephone calls & $16(6.6)$ \\
Family member questions & $15(6.2)$ \\
Patient call lights & $44(17.9)$ \\
Miscellaneous & 244 \\
TOTAL & \\
\hline
\end{tabular}


Supplies not readily available were the third most frequently observed interruption. Some patient rooms lacked necessary nursing care items. In several cases, required equipment was not in a patient's room, and the nurse needed to go elsewhere to get it. Sometimes equipment had not been returned to its specified location following previous use. Searching for patients' medical records and acquiring and adding new pages to the medical record contributed to additional interruptions.

Phone calls were the next most frequent interruption. These included calls from other departments, calls from physicians requesting patient updates or leaving new orders, and personal phone calls from family members or friends.

Questions from family members were significant interruptions. Families either telephoned or approached the nurse directly to seek updated patient information or explanations of patient care procedures. They also asked nurses to clarify information previously received from physicians. Families frequently had questions about patients' medications. They also sought the nurse to obtain directions to other hospital departments.

The need to respond to patient call lights was a common occurrence. Patients asked for assistance to the bathroom, medication or supplies, and clarification about aspects of care.

Miscellaneous interruptions of least frequency included patients' questions during assessments, telemetry alarms, missing medications, unclear Kardex entries, intravenous alarms, correcting safety hazards, faulty equipment, calling physicians for orders, excess documents in the medical record, and working around another person.

These findings paralleled those of Potter et al. In a similar study, they identified "staff inquiries, staff communications, and equipment or resource access" ${ }^{\prime\left(p_{108}\right)}$ as the most common types of interruptions in nurses' work. Additional frequent interruption types identified in this study included internal and external phone calls to the nurse and questions from family members.

\section{Travel patterns}

Travel patterns of nurses throughout their nursing units contributed further to interruptions in workflow. Nurses traveled frequently between patient rooms, medication carts or stations, and centralized equipment and care supplies locations, responding to requests from patients and other caregivers. Nurses' patient assignments were sometimes geographically approximated but at other times were distant from each other. There were multiple entrances to nurses' stations, resulting in interweaving staff movement. Some units had "pods" or small decentralized work areas, which were used only occasionally. Patient care supplies were located in a central dispensing station, which was located at the end of units and not necessarily in close proximity to patients. Medication carts remained stationary with nurses moving between them and patients' rooms rather than nurses moving the carts along with them during medication administration.

The often disjointed traffic flow appeared to be a cause of work interruptions. Nurses were often distracted as they moved about, by changes in their focus prompted by requests from patients and other caregivers. As a result of interruptions, nurses were observed to need to recollect their thoughts and reconsider their original purpose and destination.

\section{NURSING PRACTICE IMPLICATIONS}

Meetings of nursing team members, led by the nurse team leader, at the beginning of each shift and at specified intervals throughout the shift can reduce the need for team members to spontaneously seek further information from each other. The nurse needs to regularly draw the focus of team members toward patient care to reduce personal or sideline communication during patient care activities. While casual conversation can contribute to team camaraderie, keeping it to a minimum while in front of patients reduces interruptions and conveys caring to patients. The nurse can request that members of other disciplines refrain from sideline conversations 
with nursing team members during patient care activities as well.

There are several ways that availability of supplies can be improved. Maximizing the use of "pods" or decentralized nurse workstations equipped with supplies and computers can reduce the distance nursing staff need to travel. Maintaining a controlled supply of frequently used supplies in patient rooms can limit the number of times nurses need to leave the patient's bedside during care. Returning equipment to designated locations after use and ensuring an adequate supply of necessary items can reduce interruptions for following work shifts.

Reducing interruptive telephone calls involves both limiting the number of calls and using the most effective technology for managing calls. If a unit clerk answers telephones centrally and contacts the nurse, the nurse can determine whether the call needs to be taken immediately or can be responded to later. Returning multiple calls in a planned time frame can reduce interruptions to workflow. Alternatively, a call could be redirected to another nurse or caregiver if appropriate. To manage calls that require immediate attention, the use of remote phones or communication devices is helpful. The hospital in this study employs a hands-free, telephone-based wireless device that is carried by nursing team members. This enables the nurse to communicate with team members and respond to internal and external telephone calls without leaving the bedside. Patient confidentiality can be protected by use of an earpiece that will prevent conversation from being overheard. This technology requires robust information technology support.

Questions from family members are important and need to be solicited and responded to rapidly. Questions can be anticipated and met with planned educational interventions such as scheduled family meetings. A second option is to specify one family member with whom the nursing staff can communicate. This designated family member can then share information with other family members and friends. Still a third option is for one fam- ily member to convey information to others through secure Internet. The hospital in the study uses Web-based software to enable family members to create their own Web page and share information. Information about their loved one can be accessed by designated family members and friends. This effectively reduces the number of calls from families requesting patient status reports.

Anticipating patient care needs can reduce the number of patient call light interruptions and produce greater patient satisfaction. The nursing literature contains many references to the benefits of hourly patient rounds to assess and anticipate patient needs. During hourly rounds, nursing staff can question patients about the need for pain medication, position change, toileting, and personal items. Hourly rounding can also reduce the incidence of patient falls by preventing patients from attempting to walk to the bathroom or reach care items unassisted. Nursing staff can also offer water if appropriate. Asking patients whether they have other needs before leaving the room and telling patients when the nurse will return can also reduce the number of call lights. ${ }^{17,18}$

\section{LIMITATIONS}

This study is limited in that it was conducted at only one hospital. Generalizability is limited as the system of care, operations, equipment, and staffing patterns may be unique to this hospital. All observations were made on the day shift, limiting generalizability across shifts. There may be different types and patterns of interruptions on other shifts. The nursing units displayed varying geographic areas. Two were H-shaped, 2 were T-shaped, and 2 were triangular. The unit design likely contributed to unique traffic patterns and patient assignment locations in each unit.

The observers did not enter patient rooms in an attempt to avoid creating yet another interruption or distraction; thus, some interruptions may not have been recorded. It is possible that observers classified observations into different categories, despite training by the 
researchers. Paired observation would have increased the reliability of the data.

\section{RECOMMENDATIONS FOR FUTURE RESEARCH}

Further study is needed into several areas of interruption. The timing of the interruption is probably of equal or greater importance than the type of interruption. For example, if a nurse is interrupted while programming a patient-controlled analgesia pump or while preparing a sliding scale insulin injection, a serious error can occur. ${ }^{2}$ In addition, the impact of technology on interruptions needs further investigation. Nurses now may carry cell phones and personal communication devices. These devices make the nurse more accessible; however, there is a greater chance that the nurse may be interrupted. ${ }^{19}$

Further study is needed into approaches to reduce interruptions. Because some interruptions provide useful clinical information, researchers need to determine the type of interruption that should be targeted for reduction. The sound of an alarm of a ventilator, the alarm of an infusion pump, or a call from the laboratory of a low hemoglobin and hematocrit are lifesaving interruptions. Thus, research needs to focus on reduction of the interruptions that are irrelevant to patient care. ${ }^{19}$

Brixey et $\mathrm{al}^{19}$ have recently published a concept analysis of the phenomenon of interruption. Five defining attributes were identified:
(1) a human experience; (2) an intrusion of a secondary unplanned and unexpected task; (3) discontinuity; (4) externally or internally initiated; and (5) situated within a context. Use of a conceptual model of interruptions such as this could guide the design of studies in the healthcare industry.

\section{CONCLUSION}

In an integrative literature review on safety, Tzeng and $\mathrm{Yin}^{20}$ recommended further examination of the work environment of nurses as it contributes to patient outcomes. In this study, the work environment was explored by documenting the type and frequency of interruptions and work travel patterns for nurses in medical-surgical units. The interruptions data confirmed the findings from previous studies that certain patterns of interruptions prevail for staff nurses working in medical-surgical units. Suggestions for changing delivery of care and limiting these interruptions were offered. Further study is needed to test whether any of these suggested changes are effective. Caregiver time is a precious commodity in delivering safe quality nursing care. Thus, it is important to ensure that nurses are supported by effective work systems that provide supplies, medications, equipment, and information in a fashion with limited interruptions. ${ }^{12}$ Any improvements to minimize the number of interruptions experienced by nurses will improve healthcare quality and will help ensure patient safety.

\section{REFERENCES}

1. Roche JP. A pilot study of teaching clinical decision making with the clinical educator model. $J$ Nurs Educ. 2002;41(8):365-367.

2. Beyea SC. Distractions, interruptions, and patient safety. AORN J. 2007;86(1):109-112.

3. Pizzi LT, Goldfarb NI, Nash DB. Promoting a culture of safety. AHRQ Publication No. 01-E058. http://www.ahrq.gov/clinic/ptsafety/chap40.htm. Published July 2001:chap 40. Accessed November 17, 2005.

4. Salyer R. Improving medical/surgical practice with JCAHO's 2005 National Patient Safety Goals. Nursing. 2005:12-13.
5. Clancy CM, Farquhar MB, Sharp BA. Patient safety in nursing practice. J Nurs Care Qual. 2005;20(3):193197.

6. Ebright PR, Patterson ES, Chalko BA, et al. Understanding the complexity of registered nurse work in acute care settings. J Nurs Adm. 2003;33(12):630638.

7. Heath J, Johanson W, Blake N. Healthy work environments. J Nurs Adm. 2004;34(11):524-530.

8. Potter P, Boxerman S, Wolf L, et al. Mapping the nursing process: a new approach for understanding the work of nursing. J Nurs Adm. 2004;34(2):101109. 
9. Potter P, Wolf L, Boxerman S, et al. Understanding the cognitive work of nursing in the acute care environment. J Nurs Adm. 2005;35(7/8):327335.

10. Potter P, Wolf L, Boxerman S, et al. An analysis of nurses' cognitive work: a new perspective for understanding medical errors. In: Advances in Patient Safety: From Research to Implementation. Rockville, MD: Agency for Healthcare Research and Quality; 2005. http://www.ahrq.gov/qual/advances/ htm. Published 2005. Accessed April 23, 2008.

11. Tucker AL. The impact of operational failures on hospital nurses and their patients. J Operations Manag. 2004;22:151-169.

12. Tucker AL, Spear SJ. Operational failures and interruptions in hospital nursing. Health Serv Res. 2006; 41(3):643-662.

13. Brixey JJ, Robinson DJ, Tang Z, Johnson TR, Zhang $\mathrm{J}$, Turley JP. Interruptions in workflow for RNs in a Level One Trauma Center. AMIA Annu Symp Proc. 2005:86-90.
14. Balas C, Scott LD, Rogers AE. The prevalence and nature of errors and near errors reported by hospital staff nurses. Appl Nurs Res. 2004;17(4):224-230.

15. Wolf LD, Potter P, Sledge JA, Boxerman SB, Grayson D, Evanoff B. Describing nurses' work: combining quantitative and qualitative analysis. Hum Factors. 2006;48(1):5-14.

16. Pape TM, Guerra DM, Muzquiz M, et al. Innovative approaches to reducing nurses' distractions during medication administration. J Contin Educ Nurs. 2005;36(3):108-116.

17. Assi MJ, Wilson P, Bodino J, et al. Why making the rounds makes sense. Am Nurse Today. 2008;3(2):12.

18. Meade CM, Bursell AL, Ketelsen L. Effects of nursing rounds on patients' call light use, satisfaction, and safety. Am J Nurs. 2006;106(9):58-70.

19. Brixey JJ, Robinson DJ, Johnson CW, et al. A concept analysis of the phenomenon interruption. Adv Nurs Sci. 2007;30(1):E26-E42.

20. Tzeng HM, Yin CY. No safety, no quality. J Nurs Care Qual. 2007;22(4):299-306. 